\title{
Auf halbem Weg - Erste Befunde zur ERA-Umsetzung in Baden-Württemberg
}

\author{
Reinhard Bahnmüller \\ Werner Schmidt
}

\begin{abstract}
Mit der Umsetzung der einheitlichen Entgeltrahmenabkommen (ERA) in allen Regionen der Metall- und Elektroindustrie ist eines der größten entgeltpolitischen Reformprojekte in der Nachkriegsgeschichte im Gang. Nach einem Verhandlungsmarathon, der sich über mehr als zwei Jahrzehnte erstreckte, ist es unter schwierigsten tarifpolitischen und wirtschaftlichen Rahmenbedingungen gelungen, die längst überfällige Trennung von Arbeitern und Angestellten in Entgeltfragen aufzuheben und sich auf ein einheitliches Entgeltsystem zu verständigen. Der Beitrag beschäftigt sich mit der ERA-Einführung in Baden-Württemberg, dem Bezirk, in dem im Jahr 2002 der Durchbruch in den Verhandlungen erzielt wurde. ${ }^{1}$
\end{abstract}

\section{Die Relevanz des ERA-TV}

Relevant ist der ERA-Tarifvertrag (TV) vor allem aus zwei Gründen: wegen seiner verteilungspolitischen Funktionen und Wirkungen sowie seiner Bedeutung für die Stabilität bzw. Stabilisierung des Systems der Flächentarifverträge.

Der Konflikt um die Verteilung des gesellschaftlich produzierten Reichtums wird in zwei Dimensionen ausgetragen. Die erste betrifft die Verteilungsrelationen zwischen Kapital und Arbeit, die primär im Rahmen der meist jährlichen Lohn- und Gehaltsrunden ausgehandelt werden. Die zweite bezieht sich auf die interne Verteilung zwischen den Arbeitnehmern. Dieser Konflikt findet seinen Niederschlag in Entlohnungssystemen, die festlegen, wie die unterschiedlichen Aspekte von Arbeit und Leistung honoriert werden sollen, was letztlich das Gefüge der verschiedenen „Güteklassen“von Arbeit strukturiert. Entlohnungssysteme bewerten den Wert der Arbeitskraft und konstruieren und legitimieren damit soziale Ungleichheit. In ihnen schlagen sich die Machtkonstellationen am Arbeitsmarkt ebenso nieder wie substanzielle und prozedurale Gerechtigkeitsvorstellungen.

Erklärtes Ziel des ERA ist es, das bestehende soziale Gefüge zu verändern und neu zu begründen. Die bestehenden Unterscheidungen und Ungleichbehandlungen zwischen Arbeitern und Angestellten sowie innerhalb beider Statusgruppen bei der Bewertung von Arbeit, der Vergütung von Belastungen und der Leistungsentlohnung sollen beseitigt und bei der Neube- wertung Kriterien zugrunde gelegt werden, durch die Facharbeit aufgewertet und dem Grundsatz „Gleiches Entgelt für gleichwertige Arbeit" besser entsprochen werden kann.

Bisher sind die Unterschiede zwischen den Tarifverdiensten von Angestellten und Arbeitern mit vergleichbaren Anforderungen beträchtlich. In Baden-Württemberg liegt z. B. die Facharbeiter-Ecklohngruppe (LG 7) im bisherigen Zeitlohn um mehr als $400 €$ unter der als gleichwertig geltenden Tarifgruppe der Techniker (T 2/4). Belastungen werden bisher nur im gewerblichen Bereich vergütet. Sie sind zudem an die jeweilige Lohngruppe gebunden, mit dem Effekt, dass gleiche Belastungen sehr unterschiedlich bezahlt werden. ${ }^{2}$ Erhebliche Unterschiede gibt es schließlich auch im Leistungsentgelt. In Baden-Württemberg liegt das tariflich abgesicherte Niveau im Akkord- und Prämienlohn bei $30 \%$, im Zeitlohn bei $16 \%$ und im Gehaltsbereich bei $10 \%$.

Arbeit und Leistung neu zu bewerten und dabei Veränderungen im sozialen Gefüge des Betriebes vorzunehmen, ist grundsätzlich ein schwieriges und riskantes Unternehmen. Zwar sind (relative) Abwertungen von Tätigkeiten, die es zwangsläufig geben wird, durch Absicherungsregelungen in ihren finanziellen Auswirkungen begrenzt, unerheblich sind sie gleichwohl nicht. ${ }^{3}$ Riskant ist eine Neubewertung von Arbeit und Leistung für die Gewerkschaften in der derzeitigen Situation nicht zuletzt, weil sie seit geraumer Zeit kaum mehr Verbesserungen bei den Einkommen durchsetzen konnten, sondern ihre Kraft darauf konzentrieren mussten, Verschlechterungen abzuwehren. Die Rahmenbedingungen für eine Entgeltreform sind somit schwierig.
Relevant ist ERA jedoch nicht allein wegen seiner verteilungspolitischen Funktion und Wirkungen. Mindestens ebenso

\footnotetext{
1 Dargestellt werden erste Befunde der von der Hans-Böckler-Stiftung geförderten Begleitforschung zur ERA-Umsetzung in Baden-Württemberg, die vom Forschungsinstitut für Arbeit, Technik und Kultur an der Universität Tübingen (F. A. T. K.) in Zusammenarbeit mit Hans Joachim Sperling und Martin Kuhlmann vom Soziologischen Forschungsinstitut Göttingen (SOFI) durchgeführt wird. Empirische Basis sind Expertengespräche mit Vertretern der Tarifparteien, laufende Prozessbeobachtung in mit der Umsetzung befassten Arbeitsgruppen und Gremien der IG Metall sowie Interviews mit Betriebsräten und Personalverantwortlichen in 20 Betrieben.

2 Im Tarifgebiet Südwürttemberg-Hohenzollern bekam etwa ein Beschäftigter in der Lohngruppe I mit besonders schweren Belastungen in seiner Arbeit eine Entschädigung in Höhe von 24,34 €, sein Kollege in der Lohngruppe IX erhielt dagegen für die gleiche Belastung 438,64 € (IGM BaWü/ Bildungsstätte Lohr (2004), S. 164).

3 In Baden-Württemberg ist das Risiko für diejenigen, deren Verdienst auf der Basis des ERA niedriger liegt als zuvor („Überschreiter") auf $10 \%$ des bisherigen Bruttoverdienstes begrenzt, die mit Tariferhöhungen verrechnet werden.
}

Reinhard Bahnmüller, Dr., Geschäftsführer des Forschungsinstituts für Arbeit, Technik und Kultur (F. A. T. K.) an der Universität Tübingen. Arbeitsschwerpunkte: Industriesoziologie, Industrielle Beziehungen. e-mail: reinhard.bahnmueller@unituebingen.de

Werner Schmidt, Dr., stellv. Geschäftsführer des Forschungsinstituts für Arbeit, Technik und Kultur (F. A. T. K.) an der Universität Tübingen. Arbeitsschwerpunkte: Industriesoziologie, Industrielle Beziehungen, Migrationsforschung. e-mail:w.schmidt@uni-tuebingen.de 
wichtig sind die Auswirkungen auf das System der Flächentarifverträge insgesamt, das bekanntlich seit geraumer Zeit an Auszehrung leidet (Bispinck/Bahnmüller 2007). Die Tarifbindung sinkt, und auch dort, wo Tarifverträge gelten, prägen diese die betriebliche Praxis immer weniger. Dies gilt auch für die Entgeltrahmenabkommen, wie die Tarifparteien der Metall- und Elektroindustrie übereinstimmend diagnostizieren. Die betriebliche Praxis der Entgeltfindung hat sich nicht selten weit vom Tarifvertrag entfernt, teilweise weil sich die Arbeitsaufgaben und Anforderungen mit den bisherigen Bewertungskriterien bzw. tariflichen Beispielen nur noch ungenügend erfassen lassen, mitunter weil die alten Systeme nicht mehr gepflegt und zudem inkonsequent bzw. falsch angewandt wurden. Daraus resultierten oft nicht mehr nachvollziehbare Entgeltstrukturen sowie eine Erosion der Leitfunktion von Tarifverträgen. Dem soll entgegengetreten werden. ERA soll den Betrieben wieder eine verlässliche Grundlage für die betriebliche Entgeltdifferenzierung und damit dem Flächentarifvertrag Geltung verschaffen. Misslingt dieses Vorhaben, so Südwestmetall (SWM), der zuständige Arbeitgeberverband für Baden-Württemberg, ist der Flächentarifvertrag „am Ende“. Für SWM ist die ERA-Umsetzung „die Nagelprobe auf die Zukunftsfähigkeit des Flächentarifvertrags“. Die IG Metall (IGM) zeichnet die Konsequenzen einer misslungenen ERA-Einführung zwar weniger dramatisch, geht jedoch ebenfalls davon aus, dass das Tarifsystem insgesamt erheblich geschwächt würde.

\section{Umsetzungsstand}

Die Umsetzung des ERA ist in allen Tarifregionen der Metall- und Elektroindustrie im Gang und soll bis 2008 abgeschlossen sein. Unmittelbar betroffen davon sind bundesweit ca. 2 Mio. Beschäftigte, in Baden-Württemberg sind es ca. 491.000. Vollzogen ist die Einführung dort bisher in etwa einem Viertel der Betriebe. Der Mehrheit steht die Einführung noch bevor.

Im Folgenden werden die Rolle und die Ziele der Tarifparteien im Umsetzungsprozess, zentrale Konflikte, betriebliche Umsetzungsverläufe und Effekte dargestellt. Es wird gezeigt, dass trotz vereinbarter Kostenneutralität, die eine Ausklammerung des primären Verteilungskonflikts zwischen Kapital und Arbeit sicherstellen und ein integratives Vorgehen ermöglichen sollte, der Umsetzungsprozess häufig just durch diesen Konflikt geprägt ist und sich die ERA-Einführung zu einer Auseinandersetzung zwischen den Tarifparteien entwickelt, bei der es darum geht, wessen Instrument Tarifverträge primär sind. Die Einschätzungen sind noch vorläufig, weil in der Mehrheit der Betriebe die ERA-Einführung noch nicht vollzogen ist und eine gefestigte Neuordnung, wie sich zeigt, auch nach formal vollzogener ERA-Einführung oft noch nicht gegeben ist. Dennoch zeigen sich bereits jetzt Konstellationen und Entwicklungen, die für die weitere Umsetzung prägend sein dürften.

\section{Zielsetzungen der Tarifparteien im Umsetzungsprozess}

Die Zielsetzungen der Tarifparteien liegen auf unterschiedlichen Ebenen. Verfolgt werden ordnungspolitische, verbandspolitische und entgeltpolitische Ziele.

Ordnungspolitisch geht es neben dem bereits erwähnten Ziel, ein einheitliches Bewertungssystems für alle Beschäftigtengruppen zu schaffen sowie die Facharbeit (relativ) aufzuwerten, darum, den Betrieben den Nutzen flächentariflicher Regelungen deutlich zu machen. SWM macht diesen Nutzen an drei Punkten fest:

- (Wieder)Herstellung einer strukturierten Entgeltdifferenzierung, zu der die meisten Betriebe aus eigener Kraft nicht in der Lage wären,

- Revitalisierung der Entlohnung als personalpolitisches Führungsinstrument, das durch den betrieblichen Wildwuchs über die Jahre seine Motivationswirkung verloren hat,

- Schaffung eines größeren Spielraums für die betriebliche Entgeltanpassung durch eine klare Trennung zwischen betrieblichem und tariflichem Entgelt.

Während die ersten beiden Zielsetzungen von der IGM geteilt werden, trifft dies für das dritte Ziel nicht zu. SWM geht davon aus, dass ca. $15 \%$ des bisherigen betrieblichen Entgeltvolumens tarifvertraglich nicht gedeckt sind, es sich somit um tarif- vertraglich kaschiertes, faktisch jedoch übertarifliches Entgelt handelt. Letzteres soll bei der ERA-Einführung als solches ausgewiesen und in die betriebliche Disposition gestellt werden. SWM setzt darauf, dass die betriebliche Verdienstrealität im Altsystem nicht der Papierlage des Tarifvertrags entsprach und versucht nun, mit ERA die historisch gewachsenen Strukturen aufzubrechen.

Verbandspolitisch will SWM die Entmischung von tariflichen und betrieblichen Entgeltbestandteilen nutzen, um das Verhältnis und die Verantwortlichkeiten von sektoraler und betrieblicher Ebene, Tarifverband und Mitgliedsunternehmen zu klären und neu zu justieren. Mit der ERAEinführung soll Klarheit geschaffen werden, wofür die Betriebs- und die Tarifparteien jeweils zuständig sind. Durch eine eindeutige Differenzierung zwischen tariflichen und betrieblichen Entgeltbestandteilen soll deutlich werden, dass Klagen über teure Tarifverträge unberechtigt sind und SWM nicht gewillt ist, sich die Resultate einer teils kompromissorientierten, teils auf Fehlanwendung der tariflichen Entgeltsysteme basierenden betrieblichen Entgeltpolitik ankreiden zu lassen. Mit dem Verhältnis von Betrieb und Fläche beschäftigt sich auch die IGM seit einiger Zeit - allerdings mit anderer Zielsetzung. Während die IGM darum bemüht ist, die beiden Ebenen intensiver zu verschränken, um im Prozess der Verbetrieblichung tariflicher Regelungsmaterie ihren Einfluss sichern zu können, geht es SWM um die klare Trennung beider Ebenen.

Entgeltpolitisch ist das Ziel von SWM, so wird erklärt, nicht darauf gerichtet, ERA zu einem Kosteneinsparungsprogramm zu machen, wobei allerdings hinzugefügt wird, dass sich durch eine tarifkonforme Umsetzung durchaus mittelfristig Kostensenkungen ergeben könnten. Primär geht es SWM darum, besagte $15 \%$ des bisherigen Entgeltes als übertarifliches auszuweisen. Just dies will die IGM verhindern. Ihre Zielsetzung ist es, ein möglichst hohes Eingruppierungsniveau zu erreichen und das bisherige Entgeltvolumen dauerhaft, am besten ergänzungstarifvertraglich zu sichern. Darüber hinausgehende Ziele, etwa mehr „Unterschreiter" (Beschäftigte deren Verdienst mit ERA steigt) als „Überschreiter“ (deren Entgelt ohne Absicherung nach ERA geringer ausfällt) oder eine Erhöhung der betrieblichen Entgeltlinie, um die im Vorfeld rechnerisch ermittelte Größe von 2,79 \% zu 
erreichen, werden nicht als Vorgabe für alle Betriebe kommuniziert. Insgesamt haben die entgeltpolitischen Zielsetzungen der IGM einen deutlich defensiven Charakter und sind vor allem darauf gerichtet, das bisher bestehende Niveau zu halten.

Anders als beim Grundentgelt, wo SWM den Spielraum für die Betriebe möglichst klein, die IGM möglichst groß halten will, liegen die Interessenlagen beim Leistungsentgelt. Der ERA-TV sieht die Aufhebung der Unterscheidung zwischen Zeitund Leistungslohn sowie Gehalt und die Einführung eines für alle einheitlichen (ca.) $15 \%$-igen Leistungsanteils im Durchschnitt aller Beschäftigten vor. Zulässig sind drei Methoden, die auch in Kombination angewandt werden können: Kennzahlenvergleich (bisher Akkord/Prämie), Beurteilungenen und (neu) Zielvereinbarungen. Hier will SWM keine „vorkonfektionierten Lösungen“, vielmehr soll „so gut wie alles möglich sein" (SWM o. J.a), wobei jedoch sorgsam darauf geachtet werden soll, dass die Leistungsgrade sich nicht von im betrieblichen Durchschnitt zu erreichenden $15 \%$ Leistungsentgelt entfernen. Deshalb stellt der Verband Systeme bereit, die eine „automatische" Rückkehr auf diese Größe sicherstellen. Was die Methoden der Leistungsbewertung angeht, soll jedoch Wahlfreiheit herrschen. Letzteres ist nun wiederum nicht im Interesse der IGM, die darauf drängt, der Methode Kennzahlenvergleich Priorität zu geben und die Kombinationsmöglichkeiten einzuschränken. Auch hat sie kein Interesse daran, den $15 \%$ Durchschnitt festzuschreiben. Liegt die Leistung höher, müsse sich das auch im Leistungsgrad niederschlagen können. Erwartungsgemäß unterschiedlich sind die Zielsetzungen bezogen auf die Vergütung von Belastungen. Während SWM darauf orientiert, möglichst viel als „mittlere “ Belastung auszuweisen, da diese bereits im Grundentgelt abgegolten ist, und darüber hinausgehende Belastungsstufen zu vermeiden, ist es Ziel der IGM, die tarifvertraglich vorgesehenen Belastungsstufen auszuschöpfen.

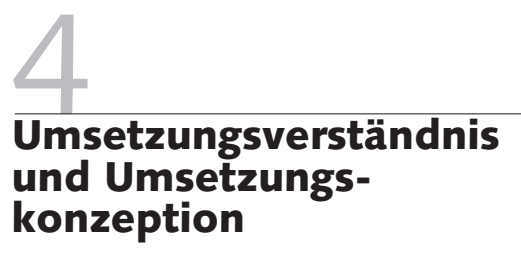

Aus den Zielsetzungen im Umsetzungsprozess ergeben sich auch die Leitlinien, auf die beide Tarifparteien die betrieblichen Akteure verpflichten wollen. Das Ziel der Entmischung tariflicher und betrieblicher Entgeltbestandteile kann SWM nur erreichen, wenn eine „Eins zu Eins Umsetzung" des ERA im eigenen Sinne erfolgt und betrieblich davon nicht abgewichen wird. SWM hält deshalb die Betriebe an der kurzen Leine und exekutiert die ERA-Umsetzung als technischen Vorgang weitgehend entpolitisierter Regelanwendung. Die Grundentgeltfindung sei im ERA-TV „abschließend geregelt, d.h. die Betriebsparteien vollziehen in der Arbeitsbewertung lediglich den Tarifvertrag". Raum für Politik gebe es deshalb nicht, sollte es zumindest nicht geben. Faktisch wird jedoch Politik im Gewande des Unpolitischen betrieben. Dem steht das Verständnis der IGM entgegen, die die Grundentgeltfindung als politischen Akt auf der Basis vereinbarter, aber deutungsoffener und konkretisierungsbedürftiger Regeln versteht. Raum für eine betriebliche Entgeltpolitik der Betriebsräte ist ihres Erachtens weiterhin gegeben und sollte auch genutzt werden.

SWM hat die eigenen Empfehlungen in einem kompakten, frühzeitig ausgearbeiteten und kommunizierten Acht-PunkteProgramm zusammengefasst. Dringend nahegelegt wird, sich so eng wie möglich an den 122 tariflichen Niveaubeispielen zu orientieren. Mit diesen seien $75 \%$ der betrieblichen Tätigkeiten abgedeckt. Weitere 20 \% könnten durch Streichen oder Hinzufügen von Teilaufgaben daraus abgeleitet werden. Lediglich für $5 \%$ der Aufgaben seien zusätzliche betriebliche Beschreibungen und Bewertungen nötig, für die SWM zudem weitere Musterbeispiele bereithält (75-20-5 Regel). Beschreibung und Bewertung der Arbeitsaufgabe sollten grundsätzlich ohne Betriebsrat vorgenommen werden. Um Bereichsopportunismus zu verhindern, sollen Führungskräfte in die Beschreibung, nicht aber in die Bewertung einbezogen werden. Alle Beschreibungen und Bewertungen sollten SWM zur Überprüfung übergeben werden.

Die Empfehlungen der IGM verhalten sich dazu spiegelbildlich (IG Metall BaWü 2005). Die wesentlichen Punkte sind:

- Klärung der Eckpunkte der ERA-Einführung im Rahmen einer Betriebsvereinbarung und/oder eines Ergänzungstarifvertrags;

- Einbeziehung des Betriebsrats in die Beschreibung und Bewertung der Arbeitsauf- gabe bereits im Vorfeld der paritätischen Kommission;

- Erstellung eigener Aufgabenbeschreibungen und Bewertungen durch den Betriebsrat als Grundlage betrieblicher Verhandlungen;

- keine Einengung auf die tariflichen Niveaubeispiele, sondern Erstellung einer hinreichend großen Zahl betrieblicher Ergänzungsbeispiele, um den betrieblichen Gegebenheiten Rechnung zu tragen und den Beschäftigten eine Identifikation mit den Aufgabenbeschreibungen zu ermöglichen;

- Abbildung tatsächlich existierender und nicht fiktiver, arbeitgeberseitig gewünschter Arbeitsaufgaben in den Aufgabenbeschreibungen und Bewertungen;

- enge Abstimmung des betrieblichen Vorgehens mit der IGM.

\section{Rolle der Tarifparteien im Umsetzungsprozess}

Der ERA-Umsetzung kommt für beide Tarifparteien höchste Priorität zu, geht es dabei doch um viel, tarifpolitisch wie finanziell. Beide Tarifparteien haben deshalb einiges aufgeboten, damit ihre Zielsetzungen zum Tragen kommen. Beide wussten, dass der ERA in Baden-Württemberg eine „Zumutung“ (SWM) für die Betriebe, aber auch für die Verbände ist, weil mehr als anderswo alle Tätigkeiten und Entgeltkomponenten einem Überprüfungs- und Veränderungsdruck ausgesetzt werden. Sie versuchen deshalb mit hohem Ressourceneinsatz den Umsetzungsprozess zu steuern und die Betriebsparteien zu enger Abstimmung mit den Tarifparteien zu verpflichten. Die Beratung der Betriebsparteien wird nicht freien Beratern überlassen, sondern selbst durchgeführt. Der Beratungsansatz beider Seiten ist offensiv, wobei vor allem das Verhalten von SWM bemerkenswert ist. Gestützt auf einen Pool von mehr als 30 ERA-Beratern werden Unternehmen von SWM ,heimgesucht", Konflikte mit dem Management bzw. mit Führungskräften eingegangen und notfalls wird auch ein Rauswurf riskiert. SWM geht damit einen eigenen, organisationspolitisch durchaus riskanten Weg, von dem keineswegs sicher ist, wie weit er die Akzep$\tan z$ der Mitgliedsfirmen findet. Stil und Auftreten entsprechen jedenfalls nicht dem 
eines passiven Dienstleisters, der sich damit bescheidet, auf Kundenanforderungen zu reagieren und Beratungsangebote sowie Unterstützung auf Nachfrage zur Verfügung zu stellen, wie Haipeter/Schilling (2006) es als Modell von Gesamtmetall beschreiben. Vielmehr will SWM Kollektivinteressen der Unternehmen zur Geltung bringen und ist dabei nicht nur weiterhin einer „Einflusslogik“ verpflichtet (Streeck 1999), sondern versucht darüber hinaus ihr Profil zu schärfen und ihre Rolle neu zu definieren. Ob SWM dies durchhalten kann und organisationspolitisch gestärkt aus der ERA-Umsetzung herausgehen wird, muss sich erst noch zeigen. Erreicht hat der Verband mit seinem offensiven Vorgehen und seinem schlagkräftigen Beraterpool, dass die Initiative eindeutig auf Arbeitgeberseite liegt. Es ist dem Verband in hohem Maße gelungen, die Deutungshoheit über die Auslegung des Tarifvertrags $\mathrm{zu}$ gewinnen, ein Terrain, das traditionell von der IGM und den Betriebsräten beherrscht wurde.

SWM praktiziert nicht nur ein anderes Rollenverständnis, der Verband positioniert sich auch gegenüber dem Flächentarifvertrag anders, als es in der Literatur als dominante Arbeitgeberverbandslinie beschrieben wird. SWM profiliert sich als grundsätzlicher Verfechter des Tarifvertrags, wohl wissend, dass „die Wertschätzung, die der Flächentarifvertrag in Baden-Württemberg hat und die Anstrengungen, ihn zu sichern, nicht von allen Schwesterverbänden gleich gesehen" werden (SWM). Der Verband ist, wie es ein Vertreter formuliert, diesbezüglich „Überzeugungstäter“. „Wir glauben daran, dass es den Betrieben wirklich nützt, dass es (den Flächentarif) gibt“. Dementsprechend wird „die Gestaltung der Arbeitsbedingungen durch Tarifverträge und die Unterstützung bei der konkreten Umsetzung in den Betrieben“ als „die Kernkompetenz von SWM“ herausgestellt (SWM o. J.b, S. 5). Eine Abkehr davon, über Kollektivverträge die Lohn- und Arbeitsbedingungen zu regeln, lässt sich zumindest für diesen Verband nicht belegen.

Bezogen auf den ERA bekommt die grundsätzlich positive Positionierung von SWM zum Flächentarifvertrag noch eine ganz besondere Wendung, weil es der Arbeitgeberverband ist, der die Ordnungsfunktion des Tarifvertrags herausstreicht und diese gegen die IGM wendet, die gemahnt wird, von den Tarifvorgaben betrieblich nicht abzuweichen. SWM kehrt somit die Rollen um, stilisiert sich selbst als die Partei, die die Flagge des Flächentarifvertrags hoch hält, während die IGM als diejenige dargestellt wird, die ihn nicht hinreichend ernst nimmt, wenn Klientelinteressen gefährdet sind. ${ }^{4}$

\section{Konfliktfelder}

Die ERA-Einführung ist reich an Konfliktfeldern. Ein zentrales sind die historisch gewachsenen betrieblichen Vergütungsstrukturen, die aufgebrochen werden sollen und deren Korrektur bzw. Überführung in die ERA-Welt vielfach zu heftigen Auseinandersetzungen führt. Dies gilt umso mehr, je weiter sich das reale betriebliche Verdienstniveau vom tariflichen Mindestniveau entfernt hat. Berührt davon sind alle Komponenten der Entlohnung.

Viele Betriebe in der Metall- und Elektroindustrie Baden-Württembergs haben traditionell ein hohes Eingruppierungsniveau, das nicht selten das tarifliche Mindestniveau überschreitet. Dafür gibt es eine Reihe von Gründen:

(1) In der Vergangenheit existierten Arbeitsmarktengpässe für bestimmte Tätigkeitssegmente, die dazu führten, dass Arbeitnehmer besser als tarifvertraglich verlangt eingruppiert wurden. Um die Motivation nicht zu gefährden, wurden $\mathrm{Ab}$ gruppierungen auch nach veränderter Arbeitsmarktlage oft nicht vollzogen.

(2) Bei arbeitsorganisatorischen Veränderungen (Re-Taylorisierung) wurde mitunter auf eine niedrigere Neubewertung der Tätigkeiten verzichtet, um die Akzeptanz der Reorganisation nicht zu gefährden.

(3) Loyalität, Seniorität oder besondere Leistungen und Verdienste wurden bisweilen mit zusätzlichen Lohn- oder Gehaltsgruppen honoriert.

(4) Viele Betriebsräte bemühten sich mit Erfolg darum, Höhergruppierungen durchzusetzen und Abgruppierungen zu vermeiden. Teilweise waren Abgruppierungen auch durch Betriebsvereinbarungen ausgeschlossen.

(5) Bei Führungskräften bestand die Tendenz, für bestimmte managementnah täti- ge Beschäftigte oberhalb des tariflich geforderten Levels zu bezahlen.

Mit der ERA-Einführung kommen die so gewachsenen Strukturen nun auf den Tisch. Sie müssen entweder korrigiert oder ERA-konform neu begründet werden, was schwierig ist und nicht immer gelingt, zumal die Betriebe häufig der Linie von SWM folgen, dass der ERA Maximal- und nicht Untergrenzen definiere. Eine „politische“ Klärung von Eingruppierungsniveaus oder zusätzliche betriebliche Entgeltbausteine sind nötig, um das bisherige Niveau halten zu können. Ohne Belegschaftsdruck und entsprechende Konflikte ist das nicht möglich.

Das Problem der gewachsenen betrieblichen Verdienststrukturen stellt sich auch beim Leistungsentgelt. Auch hier haben sich die tatsächlich erreichten und bezahlten Leistungsgrade in vielen Betrieben von dem tariflich gesicherten Mindestniveau entfernt. Deshalb kommt es zu Problemen bei der Transformation und dauerhaften Absicherung höherer Verrechnungsgrade im ERA. Hinzu kommt, dass manche Arbeitgeber beim Wechsel in den ERA auf die Methode „Kennzahlenvergleich“ (ehemals Akkord und Prämienlohn) verzichten möchten, da daran besondere Regelungen für Erholzeiten (sog. „Steinkühlerpause“) und Mitbestimmungsrechte geknüpft sind, während Betriebsräte, u.a. aus diesen Gründen, für diese Methode eintreten.

Belastungen sind ebenfalls ein betriebspolitisch heikles Thema, kreuzen sich doch auch hier "gewachsene Altstrukturen" mit der neuen Systematik des ERA und deren Handhabung durch die Arbeitgeber. Die alte tarifvertragliche Regelung sah vor, dass belastende Tätigkeiten im Arbeiterbereich $\mathrm{zu}$ höheren Lohngruppen führen. Laut ERA sind mittlere Belastungen bereits mit

\footnotetext{
4 Flächentarifverträge sind allerdings auch für SWM kein Selbstzweck, sondern Mittel zum Zweck. Bekanntermaßen hat der Verband auch andere Tarifverträge abgeschlossen, durch die die Verbindlichkeit der Tarifnormen herabgesetzt wird (Pforzheimer Abkommen). Es wird flexibel und themenbezogen geklärt, was den Interessen des Verbandes am besten entspricht. Die Leitlinie von SWM lässt sich folgendermaßen zusammenfassen: Tarifverträge nicht aufgeben, sondern teils „umnutzen" und auf ihrer Ordnungsfunktion bestehen, teils ihre bindende Wirkung mindern, versuchen, aus Mindestnormen Höchstnormen zu machen, und den "Rest" über das "Pforzheimer Abkommen" und seine Weiterentwicklung regeln.
} 
dem Grundentgelt abgedeckt, während höhere Belastungen eine von der Entgeltgruppe unabhängige, besondere Belastungszulage erbringen. Zwar ist Belastung durch Lärm klar geregelt und messbar, die Bestimmung anderer Belastungen ist jedoch umstritten. Arbeitgeberseitig wird darauf orientiert, möglichst viele Belastungen als bereits abgegoltenes „mittleres Niveau" zu bewerten. Hinzu kommt, dass die bisherige Bezahlung häufig nicht angepasst wurde, obwohl manche Belastungen durch technische Veränderungen entfallen waren. Beides zusammen führt zu einer Verkleinerung der Bereiche mit Belastungszulagen und begünstigt mitunter, dass Angelernte zu „Überschreitern“ werden.

\section{Varianten betrieblicher Einführungsprozesse}

Der Verlauf der betrieblichen Einführungsprozesse folgt keinem einheitlichen Modell. Es gibt vielfältige Varianten. Beeinflusst wird der Prozess vor allem von der Informations- und Partizipationspolitik des Arbeitgebers gegenüber Betriebsrat und Belegschaft sowie den Auf- bzw. Abwertungseffekten durch die neue Arbeitsbewertung. SWM empfiehlt den Firmen, der Kommunikation mit der Belegschaft und dem Betriebsrat viel Aufmerksamkeit zu schenken, sie aber in entscheidungsrelevante Abläufe nur in dem Maße einzubeziehen, wie es der Tarifvertrag zwingend verlangt. Aus Sicht des Verbandes kann der ERA auch „vollkommen ohne Mitwirkung des Betriebsrats" eingeführt werden (SWM 2005, S. 12), was zwar nicht generell empfohlen, aber als zulässige Möglichkeit offeriert wird. Nicht wenige Firmen sind der minimalistischen Beteiligungslinie gefolgt, allerdings mit meist konfliktreichen Folgen.

\subsection{EINSEITIGES ARBEITGEBERHANDELN}

Häufig findet sich ein einseitiges Vorgehen der Arbeitgeber. Beschreibung und Erstbewertung der Arbeitsaufgaben werden ohne Einbeziehung des Betriebsrats vorgenommen und der Paritätischen Kommission übergeben, ohne dass ersichtlich wird, welche Personen bzw. konkreten Arbeitsplätze bewertet wurden. Letzteres wird erst bekannt, wenn die Mitteilung der Arbeitsbewertung an die Beschäftigten erfolgt, oft erst unmittelbar vor dem arbeitgeberseitig festgelegten Einführungszeitpunkt. Der Prozess wird dann betriebsöffentlich, der Arbeitgeber muss die Hinterbühne verlassen (Friedman 1994). Konfrontiert mit den erfolgten Eingruppierungen, die häufig nicht den Erwartungen der Beschäftigten und der Betriebsräte entsprechen, leiten sie Gegenmaßnahmen ein. Es kommt zu einer Vielzahl an Reklamationen, vereinzelt werden alle Bewertungen reklamiert. Die Betriebsräte können diesen Unmut nutzen, um mit Nachdruck auf Korrekturen zu drängen. Auch manche Vorgesetzte fürchten jetzt demotivierte Mitarbeiter und setzen sich für Nachbesserungen ein. Welches Ausmaß die Konflikte erreichen, hängt von der Unzufriedenheit der Beschäftigten mit ihren Bewertungen, der Konfliktfähigkeit und -bereitschaft der Betriebsräte und vom Entgegenkommen des Arbeitgebers ab. Hier gibt es Unterschiede zwischen den Betrieben dieses Typs. Gemeinsam ist ihnen jedoch, dass die eigentliche Auseinandersetzung und die relevanten Aushandlungsprozesse nicht vor, sondern nach der ERAEinführung stattfinden. Meist erreichen die Betriebsräte mit Unterstützung der Belegschaft im Nachgang relevante Verbesserungen, die Auseinandersetzungen über die tatsächlichen Bewertungen und Eingruppierungen ziehen sich jedoch über Monate hin.

\subsection{ERWEITERTE KOOPERATION}

Die Arbeitgeber größerer Betriebe (aber nicht nur dort) bevorzugen nicht selten bereits in der Vorphase der ERA-Einführung eine stärkere Konsultation der Betriebsräte. Über das zwingend vorgeschriebene Maß an Mitwirkung hinaus werden gemeinsame Projektgruppen von Personalwesen und Interessenvertretung gebildet, die Prozeduren der ERA-Einführung diskutiert, Betriebsvereinbarungen formuliert und teilweise auch eine größere Zahl betrieblicher Ergänzungsbeispiele vereinbart. Interessendivergenzen werden bereits im Vorfeld ausgetragen und den ERA ergänzende Vereinbarungen getroffen. So werden mitunter der $10 \%$-ige Anrechnungsbetrag für „Überschreiter“ reduziert, zusätzliche betriebliche Entgeltbausteine konzipiert oder bereits vor Beginn der Arbeit der Paritätischen Kommission politische Klärungen zur Einstufung bestimmter Arbeitsaufgaben herbeigeführt (etwa in Fertigung und Montage), um deren Grundentgeltniveau langfristig zu sichern. Bisweilen erklären sich Betriebsräte auch dazu bereit, auf Mittel des ERA-Anpassungsfonds zu verzichten, um unter Managementdruck und/ oder im Kontext von Standortvereinbarungen Arbeitsplätze zu sichern.

Eine über das (umstrittene) tarifliche Mindestmaß hinausgehende Beteiligung des Betriebsrats und im Vorfeld getroffene Vereinbarungen reduzieren die Wahrscheinlichkeit späterer Konflikte, sie bewahren jedoch nicht davor. Erstens schützen auch zusätzliche Regelungen nicht vor einem verbleibenden Interpretationsspielraum. Zweitens wird die Zuordnung einzelner Arbeitsplätze zu den Beispielen den Betriebsräten auch in diesen Betrieben nicht immer vor der Mitteilung an die Beschäftigten bekannt. Drittens greifen auch hier die Beschäftigten erst nach dem Erhalt der Mitteilungen selbst als aktive Teilnehmer in das Geschehen ein. Die ERA-Einführung verläuft somit auch dann selten konfliktfrei, wenn partiell zwar die Betriebsräte in die Diskussion der Beispiele einbezogen werden, jedoch die Zuordnung der tatsächlichen Tätigkeiten zu den Beispielsfällen im Unklaren bleibt.

\subsection{HOHE KOOPERATION}

Durchgehend kooperative Einführungsprozesse scheinen eher die Ausnahme zu sein, finden sich allerdings auch in den Untersuchungsbetrieben. Bei den Betrieben, die wir diesem Typ zuordnen, sind unterschiedliche Gründe hierfür maßgeblich. In manchen Betrieben entspricht es der Tradition des Hauses, einen kooperativen Umgang mit dem Betriebsrat zu pflegen. Von Beginn bis Ende wird das ERA-Projekt gemeinsam betrieben, bereits vor der Bildung der Paritätischen Kommission wird paritätisch gearbeitet. Schulungen der Vorgesetzten werden gemeinsam durchgeführt, Arbeitsbeschreibungen und Bewertungen zusammen erstellt bzw. frühzeitig ausgetauscht, alle Mitarbeiter in Gruppen- und Einzelgesprächen vor Einführung des ERA informiert. Betriebsrat und Beschäftigte sind über den jeweils aktuellen Bearbeitungsstand informiert, größere Überraschungen im Zuge der Mitteilungen an die Beschäftigten gibt es deshalb nicht. Entsprechend gering sind die Reklamationsquoten. 
Mitunter sind betriebliche Sonderbedingungen für die durchgehend kooperative ERA-Einführung maßgeblich. In einem Betrieb etwa, der bis zur ERA-Einführung keiner Tarifbindung unterlag, hatte sich im Verlauf etlicher Jahre eine hochgradig unsystematische Bezahlungspraxis entwickelt, die den Arbeitgeber dazu bringt, den ERA zu übernehmen und dem Arbeitgeberverband beizutreten. Die Eingruppierung wird von den Betriebsparteien gemeinsam erarbeitet, praktiziert wird eine „integrative“ ERA-Umsetzung (Walton/ McKersie 1965), beide können eine Verbesserung im eigenen Sinne erreichen und gemeinsam gegenüber der Belegschaft vertreten. Ein anderer Betrieb ist mit einer Kostensenkungsvorgabe der Konzernzentrale konfrontiert, es stellt sich die Alternative Personalabbau oder Kostensenkung. Auf einer Belegschaftsversammlung wird zugestimmt, ERA frühzeitig einzuführen, die Arbeitsbeschreibungen und Bewertungen nach ERA gemeinsam und ohne Blick auf das bisherige Verdienstniveau vorzunehmen und auf den Ausgleichsbetrag für „Überschreiter" sofort zu verzichten. Zwar gibt es Unmut in besonders negativ betroffenen Abteilungen, die Mehrheit der Belegschaft und der Betriebsrat ziehen eine kooperative Mitwirkung bei der kostensparenden ERA-Einführung jedoch drohenden Entlassungen vor.

\section{Materielle Effekte und Beschäftigtenreaktionen}

Die Tarifparteien nahmen an, durch die Einführung des ERA würde sich die betriebliche Entgeltsumme im Durchschnitt der Betriebe um 2,79 \% erhöhen. Für fünf Jahre ist den Betrieben dennoch Kostenneutralität garantiert. Dazu wurden in den Tarifrunden Rückstellungen aus nicht ausbezahltem Entgelt vorgenommen, die in den ERA-Anpassungsfonds fließen. Bei Überschreitung der 2,79\% in dem FünfJahres-Zeitraum, sind weitere Ausgleichsmechanismen vorgesehen. Umgekehrt darf die Entgeltsumme der Betriebe durch die ERA-Einführung auch nicht unter das bisherige Niveau fallen. Käme es dazu, wären ebenfalls Ausgleichszahlungen fällig. Derzeit spricht wenig dafür, dass die rechnerisch ermittelten 2,79\% an Mehrkosten erreicht werden. Die von uns untersuchten
Betriebe erzielen diese Steigerung größtenteils nicht, erste Auswertungen der Betriebsrätebefragung in Betrieben, die den ERA eingeführt haben, bestätigen diesen Befund. Zumindest langfristig werden sich deshalb für die Arbeitgeber Kostensenkungseffekte ergeben.

Welche Beschäftigtengruppen strukturell zu den Gewinnern („Unterschreiter") und den Verlierern („Überschreiter") der ERA-Einführung gehören werden, lässt sich derzeit nur sehr vorläufig beantworten. In der Tendenz zeigt sich, dass Facharbeitertätigkeiten in der Produktion und in den produktionsnahen Bereichen - wie intendiert - höher bewertet werden. Den Aufwertungen stehen allerdings Abwertungen auch bei diesen Tätigkeiten in nicht geringer Zahl gegenüber. Die ERAUmsetzung ist auch durch einen Streit geprägt, was zukünftig unter Facharbeit $\mathrm{zu}$ verstehen ist. Angelernte Beschäftigte im ehemaligen Leistungslohn, (einfache) kaufmännische, Sekretariats- und Verwaltungstätigkeiten sowie technische Beschäftigtengruppen, soweit ehemals besondere Arbeitsmarktengpässe bestanden, scheinen hingegen eindeutig zu den strukturellen Verlierern der ERA-Einführung zu gehören. Hier konzentrieren sich die „Überschreiter" und auch die Reklamationen. Wie das Verhältnis von „Unterschreitern“ und „Überschreitern“ letztlich sein wird, lässt sich derzeit noch nicht sagen, in einer ganze Reihe der von uns untersuchten Betriebe liegt jedoch der Anteil Letzterer höher.

Da gleichwohl nur ein Teil der Beschäftigten zu den „Überschreitern“ zählt, das Verdienstrisiko durch tarifliche Absicherungen begrenzt und teilweise durch betriebliche Regelungen weiter verringert ist, könnte angenommen werden, dass sich die Aufregung unter den Beschäftigten in Grenzen hält. In der Regel ist das nicht der Fall. Dabei macht sich der Unmut nicht nur an Verdiensteinbußen fest. Es geht nicht zuletzt um Fragen der Anerkennung. Selbst Beschäftigte, deren Entgelt vollständig gesichert ist, empören sich, so wird häufig berichtet, über mangelnde Wertschätzung im Zuge der ERA-Einführung. Mehrere Gründe spielen dabei eine Rolle. Erstens signalisiert die Zugehörigkeit zu den „Überschreitern", man erhielte sein Entgelt bisher zu Unrecht und zukünftig nicht auf der Basis eines anerkannten Beitrags. Zweitens führt die Zuordnung zur bisher nicht besetzten Entgeltgruppe 1 zur Wahrneh- mung, man sei jetzt "ganz unten“ angekommen. Drittens finden Beschäftigte ihre tatsächliche Tätigkeit in den nunmehr abstrakteren Aufgabenbeschreibungen nicht oder nur partiell wieder und interpretieren dies als Missachtung ihrer real geleisteten Arbeit. Viertens wird es als Missachtung der beruflichen Identität verstanden, wenn Ingenieure einem Technikerbeispiel oder Techniker einem Facharbeiterbeispiel zugeordnet werden. Sensibel sind solche Zuordnungen selbst dann, wenn die betroffenen Beschäftigten nach ERA mehr verdienen als zuvor. Bemerkenswerterweise erweist sich die Bedeutung der Anerkennungsfrage für das Feld der industriellen Beziehungen (Schmidt 2005) damit gerade beim „harten“ Thema Entgelt.

\section{Resümee}

Sowohl beim Grundentgelt (frühere Eingruppierung häufig oberhalb des Mindestniveaus), beim Leistungsentgelt (hohe Akkord- oder Prämiensätze) als auch bei den Belastungen (Fortzahlung trotz entfallener Belastungen) zeigt sich somit, dass die positive Abweichung der realen, gleichwohl als tariflich gesichert geltenden Lohn- und Gehaltsstrukturen von den tariflichen Mindestbestimmungen ein zentrales Problem der ERA-Einführung ist. Für die IGM ist dies aus zwei Gründen brisant: Erstens ist die Abweichung der Altstrukturen von der tariflichen Papierlage vor allem in Betrieben groß, in denen der gewerkschaftliche Organisationsgrad überdurchschnittlich ist und Betriebsräte eine aktive betriebliche Entgeltpolitik betrieben haben. Letztere erleben die Umstellung auf den ERA deshalb teilweise auch als eine Entwertung ihrer bisherigen Arbeit. Sie machen hierfür nicht nur den Arbeitgeber, sondern auch die Gewerkschaft verantwortlich. Zweitens ist der Anteil der „Überschreiter" sowohl in gewerkschaftlich gut organisierten Bereichen der klassischen Leistungslöhner, also in den bisherigen gewerkschaftlichen Kampfbereichen, als auch bei Beschäftigtengruppen hoch, die bisher zwar selten organisiert sind, die jedoch zu einem zukünftig wichtigen Organisationsfeld zählen. Das Risiko der IGM besteht darin, in ihren bisherigen Bastionen Rückhalt zu verlieren, ohne bei ihrer Zukunftsklientel entsprechend zu gewinnen. Zwar 
haben sich Befürchtungen, ERA könnte zu massiven Mitgliederverlusten führen, bisher ebenso wenig bestätigt wie Hoffnungen, ERA würde sich zu einem Zugpferd der Mitgliederrekrutierung entwickeln. Doch einen Imagegewinn beim Gros der Beschäftigten kann die IGM auch nicht verzeichnen. Noch dominiert die Kritik. Allerdings scheint dies für alle beteiligten Akteure zu gelten: Management, Betriebsräte, Arbeitgeberverband und Gewerkschaft.

Das offensive Vorgehen von SWM, also die Rollenumkehr zwischen den Tarifparteien und der bisher nicht gekannte Hegemonieanspruch von SWM bei der Tarifvertragsauslegung, kam für die IGM überraschend und führte zu spürbarer Verunsicherung in deren Reihen. Es hat geraume Zeit gebraucht, bis sich die IGM und die Betriebsräte darauf eingestellt hatten und fortan durch eine offensivere Gangart versuchen, die zwischen den Tarifparteien strittigen und nicht lösbaren Fragen auf betrieblicher Ebene und unter Einschluss von
Protestaktionen zu klären. Wie sich zeigt, geht es bei der ERA-Einführung nicht nur um eine Neubestimmung der Binnendifferenzierung zwischen den Beschäftigten. Es geht auch um einen Verteilungskonflikt zwischen Kapital und Arbeit und nicht zuletzt darum, wessen Instrument der Flächentarifvertrag zukünftig primär sein wird. SWM exerziert am Beispiel des ERA vor, dass Verteilungspolitik sich auch im Gewand von Ordnungspolitik betreiben lässt und eine Abkehr vom Flächentarifvertrag nicht das Credo einer den Interessen der Arbeitgeber entsprechenden Politik sein muss. Tarifverträge, richtig konzipiert und konsequent umgesetzt, so lautet die Botschaft auch in die eigenen Verbandsreihen, sind von unmittelbarem Nutzen für die Arbeitgeber. Zur Disposition steht für SWM derzeit nicht der Flächentarifvertrag generell, sondern sein interessenpolitischer Gehalt. Tarifverträge nicht aufgeben, sondern „umnutzen“, sie von einem Instrument, das traditionell primär der Durchsetzung von Arbeitnehmerinteressen dient, zu einem zu machen, bei dem die Interessen der Arbeitgeber stärker zum Tragen kommen, so könnte die Linie des Verbandes kurz zusammengefasst werden. Eine solche Veränderung entspräche dem, was Streeck/Thelen (2005) mit dem Begriff der „Konversion“ von Institutionen beschreiben. Eine solche Konversion kann nicht weniger konsequenzenreich sein als eine Aushöhlung oder ein Zusammenbruch. Unseres Erachtens kann die ERA-Umsetzung als Versuch einer solchen Konversion der Institution Flächentarifvertrag gedeutet werden. Auch weil es über den ERA hinaus in der Metall- und Elektroindustrie wie in anderen Branchen weitere Anhaltspunkte in diese Richtung gibt, scheint es uns aus theoretischer wie aus politischpraktischer Sicht ratsam, den Wandel der Institution Flächentarifvertrag nicht nur in Begriffen wie „Aushöhlung“ oder „Deregulierung" zu beschreiben, sondern interessenpolitischen Umnutzungen mehr Beachtung zu schenken.

\section{LITERATUR}

Bispinck, R./Bahnmüller, R. (2007): Abschied vom Flächentarifvertrag? Der Umbruch der deutschen Tariflandschaft und seine Konsequenzen für eine betriebsorientierte Tarifpolitik, in: Bispinck, R. (Hrsg.): Wohin treibt das Tarifsystem?, Hamburg, S. 9-28

Friedman, R. A. (1994): Front Stage, Backstage. The Dramatic Structure of Labor Negotiations, Cambridge, Mass./London

Haipeter, T./Schilling, G. (2006): Von der Einfluss- zur Mitgliedschaftslogik. Die Arbeitgeberverbände und das System der industriellen Beziehungen in der Metallindustrie, in: Industrielle Beziehungen 13, S. 21-42 IG Metall BaWü/Bildungsstätte Lohr (2004): ERA-Wissen Handbuch I. Arbeitsbewertung und Arbeitsbelastung, Lohr IG Metall BaWü (2005): Handlungshilfe ERA-Einführungsprozess, Stuttgart

Schmidt, W. (2005): Industrielle Beziehungen, Interesse und Anerkennung. Plädoyer für eine duale Perspektive, in: Industrielle Beziehungen 12 , S. $51-73$
Streeck, W. (1999): Staat und Verbände: Neue Fragen, neue Antworten?, in: Ders. (Hrsg.): Korporatismus in Deutschland. Zwischen Nationalstaat und Europäischer Union, Frankfurt/Main und New York

Streeck, W./Thelen, K. (2005): Introduction, in: Diess. (Hrsg.): Beyond Continuity. Institutional Change in Advanced Political Economies, Oxford, S. 1-39

Südwestmetall (SWM) (2005): Erläuterungen. Einführungstarifvertrag zum ERA-TV für die Beschäftigten der Metall- und Elektroindustrie in Baden-Württemberg, 1. Auflage, September, Stuttgart

Südwestmetall (SWM) (o. J.a): ERA-Infobrief 1-3, Stuttgart Südwestmetall (SWM) (o. J.b): Was Unternehmen bindet, Stuttgart Walton, R. E./McKersie, R. B. (1965): A Behavioral Theory of Labor Negotiations. An Analysis of a Social Interaction System, New York 